\title{
Theoretical Study of Double Transition in Specific Heat of Filled Skutterudite $\mathrm{PrOs}_{4} \mathrm{Sb}_{12}$
}

\author{
Abid Boudiar (Corresponding author) \\ Laboratoire de physique théorique et appliquée, Université de Tebessa, Algeria \\ E-mail: boudiar_a@yahoo.fr
}

Samir Khéne

Département de Physique, Faculté des Sciences, Université Badji Mokhtar d'Annaba

Bp 12, 23000, Annaba, Algerie

E-mail: samikhen@yahoo.fr

\begin{abstract}
The specific heat is found with multiple phase transition like huge and sharp jumps in narrow interval of low temperature. We found a big value of the electronic specific heat coefficient. Results of our calculations are in agreement with experimental data for the filled Skutterudite compound $\mathrm{PrOs}_{4} \mathrm{Sb}_{12}$.
\end{abstract}

Keywords: Specific heat, Multiple phase transition, Filled Skutterudite, $\mathrm{PrOs}_{4} \mathrm{Sb}_{12}$

\section{Introduction}

Skutterudite compound has received considerable attention during the past years. Care by researchers because it is a good thermoelectric material, for this has been a number of experiments about this property. Also been many theoretical studies of the electronic structure. The Pr-based filled-skutterudite compounds have attracted much attention because of their exotic properties like metal-insulator transition or unusual heavy-fermions behavior (C. Sekyne, 1997; H. Sato, 2000; H. Sugawara, 2002).This also concern recently discovered $\mathrm{PrOs}_{4} \mathrm{Sb}_{12}$, the first known example of a heavy -fermions superconductor containing Pr .heavy-fermions behavior is inferred by the size of the jump in the heat capacity at $\mathrm{T}_{\mathrm{c}} \sim 1.76 \mathrm{k}^{\circ}$ and by the slope of the critical field near $\mathrm{T}_{\mathrm{c}}$. Both analyses suggest $\gamma \approx 350 \mathrm{~mJ} / \mathrm{mol}^{2} \mathrm{k}^{2}$ and $m^{*} \approx 100 m_{e}$ (K. A. Gschneider, 2003). In 2003 Vollmer and al (R. Vollmer, 2003), Maple and al (M.B. Maple, 2003), Relate the existence of double transition in the specific heat of $\mathrm{PrOs}_{4} \mathrm{Sb}_{12}$ nearby $\mathrm{T}_{\mathrm{c}}$ about $\mathrm{T}_{\mathrm{c} 1} \sim 1.82 \mathrm{k}^{\circ}$ and $\mathrm{T}_{\mathrm{c} 2} \sim 1.74 \mathrm{k}^{\circ}$ with a jumps of equivalent height. This double transition has since been observed repeatedly by several research groups (K. Izawa, 2003; N. Oeschler, 2003; N. Oeschler, 2004). This confirmed the existence of two phases of superconductivity with different parameter. In this work we suggest theoretical study of double transition in specific heat of filled Skutterudite $\mathrm{PrOs}_{4} \mathrm{Sb}_{12}$, for that we consider a quasiparticiles with many configuration of spin interact via a weak screened interaction. Starting with phenomenological (M. Sigrist, 1991) functional energy which used to found specific heat. We adjust our parameters to fit the experimental data of Skutterdudite compound $\mathrm{PrOs}_{4} \mathrm{Sb}_{12}$.In the latter we compare our results with the empirical data.

\section{Calculus}

Because the quasiparticles are long lived at low energies, and have the same internal quantum numbers as bare electrons (P. Gegenwart, 2008), the functional energy is defined from phenomenological point view as follows:

$$
\varepsilon_{n}(\alpha, T)=\frac{\varepsilon_{0}}{(\alpha-1)^{2}}(n+\alpha-1)^{2}+\theta\left(\varepsilon_{n}-\varepsilon_{1}\right) \eta(\alpha) T
$$

Where $\alpha$ and $\eta$ are some phenomenological expansion coefficients which are characteristics of the material. $\theta$ Is the Heaviside step function, $T$ the temperature, $n \in N$ and $\varepsilon_{n+1} \succ \varepsilon_{n} \succ 0$. It will be easy to understand the physical significance of functional energy $\varepsilon_{n}(\alpha)$ if we reduced it to a simple form:

$$
\varepsilon_{n}=\varepsilon_{0}^{0}+\varepsilon_{n}^{h}+\varepsilon_{n}^{f}+\varepsilon_{n}^{T}
$$


$\varepsilon_{n}^{h}$ : is the energy of harmonic oscillator which represented the effective interaction (E. A. Goremychkin, 2004).

$$
\varepsilon_{n}^{h}(\alpha)=\frac{2 \varepsilon_{0}}{(\alpha-1)}\left(n+\frac{3}{2}\right)
$$

$\varepsilon_{n}^{f}$ : As kinetic energy of free particle in box. The effective interaction between quasiparticles is screened and considerably weaker than the bare colomb interaction between electrons. In fact, the screened interaction is sufficiently small so that quasiparticles can be regarded as approximately independent, which finally justifies the independent-particle approximation and explains the success of mean -field theories (C. Friedrich, 2006).

$$
\varepsilon_{n}^{f}(\alpha)=\frac{\varepsilon_{0}}{(\alpha-1)^{2}} n^{2}
$$

We can see two opposite work: entropy to make electrons free and effective interaction to make it in quasiparticules, where $(\alpha-1)^{-1}$ plays the role of coupling constant.

$$
\varepsilon_{0}^{0}(\alpha)=\frac{\alpha-4}{\alpha-1} \varepsilon_{0}
$$

$\varepsilon_{0}^{0}$ : is the ground state energy, to give minimum values for $\varepsilon_{n}(\alpha)$, we take $1 \prec \alpha \leq 4$.

There is degeneration $g_{n}=2(n-1)$ in functional energy for the spin orientation (see table 1), after some calculations and using variable change $T T_{c}^{-1}=\mu x$, the partition functional can be written as:

$$
\begin{aligned}
& Z=Z_{0}^{X}\left\{\alpha^{2}\left[1-2 \sum_{p=1}^{\infty}(2 p-1) \frac{B_{2 p}}{(2 p) !}\left(\frac{1}{\alpha^{2} x}\right)^{p} H_{2(p-1)}\left(\frac{1}{\sqrt{x}}\right)\right] e^{\frac{-1}{x}}\right. \\
& \left.+\alpha^{2} \sqrt{\pi x^{-1}}\left[-1+e r f\left(\frac{1}{\sqrt{x}}\right)\right]\right\}^{\mu x}
\end{aligned}
$$

$\mathrm{H}_{2(\mathrm{p}-1)}$ is Hermit polynomial, $\mathrm{B}_{2 \mathrm{p}}$ is Bernoulli numbers, $\mathrm{Z}_{0}$ is function in $\mu, \eta$ and $T_{C}$.

Where $\mu$ is parameter given by:

$$
\mu=\frac{\varepsilon_{0}}{K_{B} T_{C}}\left(\frac{\alpha}{\alpha-1}\right)^{2}
$$

\section{Results and discussion}

Specific heat is plotted in Figure. 1 for $\mu \approx 34,48276$, the results of the simulation are compared in Table. 2 with available experimental data of $\mathrm{PrOs}_{4} \mathrm{Sb}_{12}$, witch measured by Vollmer (2003), and Maple (2003), where the specific heat unit is $\left(\mathrm{j} \cdot \mathrm{mol}^{-1} \cdot \mathrm{k}^{-1}\right), \gamma_{e l}$ in $\left(\mathrm{mj}^{\mathrm{m}} \mathrm{mol}^{-1} \cdot \mathrm{k}^{-2}\right)$ and $\Delta T=T_{c 1}-T_{c 2}$. Good agreement exists between the results of our numerical calculations and the experimental data. Simulations gave an other multiple phase transition like huge and sharp jumps in narrow interval of low temperature when passing from normal state to the superconductivity state before $T_{c 1}$ (see figure2). Previous transitions may be present in reality but the experiment did not reveal it because narrow range of temperatures and the quick change in specific heat.

\section{References}

C. Friedrich and A. Schindlmayr. (2006). NIC. Series. 31, 335-355.

C. Sekyne, T. Uchiumi, I. Shirotani, and T. Yagi. (1997). Phys. Rev. Lett. 79, 3218.

E. A. Goremychkin, R. Osborn, E. D. Bauer, and M. B. Maple, N. A.Fredderick, W. M. Yuhsz, F. M. Woodward, 
J. W. Lynn. (2004). Phys. Rev. Lett. 93, 157003.

H. Sato, Y. Abe, H. Okada, T.D.Matsuda, K.Abe, H. Sugawara, and Y. Aoki. (2000). Phys. Rev. B 62, 15125 .

H. Sugawara, T. D. Matsuda, K. Abe, Y. Aoki, H. Sato, S. Nojiri, Y. Inada, R. Settai, and Y. Onuki. (2002). Phys. Rev. B 66, 134411 .

K. A. Gschneider, Jr., J.-C. G. Bunzil, and V. K. Pecharsky, W. (2003). Handbook on the Physics and chemistry of Rare earths. The Holland: Elsevier, (Volume33, P22).

K. Izawa, Y. Nakajima, J. Goryo, Y. Matsuda, S. Osaki, H. Sugawara, H. Sato, and P. Thalmeier, K. Maki. (2003). Phys. Rev. Lett. 90, 117001.

M. Sigrist, and K. Ueda. (1991). Rev. of Modern Phys. 63, 239.

M.B. Maple, Ho,-P.-C, Zapf,-V.-S., N.A. Frederick, E.D. Bauer, W.M. Yuhasz, and F.M. Woodward, J.W. Lynn. (2003). J. Phys. Sos. Of Jpn. 71, Suppl. 23.

N. Oeschler, P. Gegenwart, and F. Stegglich, N.A. Frederick, E.D. Bauer, M.B. Maple. (2003). Acta Phys. Pol. B 34,959 .

N. Oeschler, P. Gegenwart, F. Weickert, I. Zerec, P. Thalmier and F. Stegglich, M.B. Maple. (2004). Phys. Rev. B 69, 235108.

P. Gegenwart, Qimiao Si and F. Steglich. (2008). Nature. Physics. 4, 188.

R. Vollmer and A. Faifst, C. Pfleiderer, H. v. Lohneysen, E.D. Bauer, P.-C.Ho, V.Zapf and M.B. Maple. (2003). Phys. Rev. Lett. 90, 057001.

Table 1. The quasipartcules for $n=2,3$ and 4 . We use ( + ) and (-) for spins (up) and (down)

\begin{tabular}{ccc}
\hline $\mathrm{n}$ & $\mathrm{g}_{\mathrm{n}}$ & quasiparticles \\
\hline 2 & 2 & $(++),(+-)$ \\
3 & 4 & $(+++),(++-),(+-+),(--+)$ \\
4 & 6 & $(++++),(+++-),(++--),(+--),(++-+),(+--+)$ \\
\hline
\end{tabular}

Table 2. Comparison our numerical simulations with experimental data [6] of $\mathrm{PrOs}_{4} \mathrm{Sb}_{12}$

\begin{tabular}{ccc}
\hline Results & Exp & The \\
\hline$\Delta T / T_{,}$ & 0.045977 & 0.035087 \\
$T, / T_{1}$ & 0.956044 & 0.966102 \\
$\gamma_{o l}$ & 350 & 378.1517 \\
$C_{o l}^{2}$ & 2.1 & 1.7728 \\
$C_{e l}^{1}$ & 1.4 & 1.1634 \\
\hline
\end{tabular}




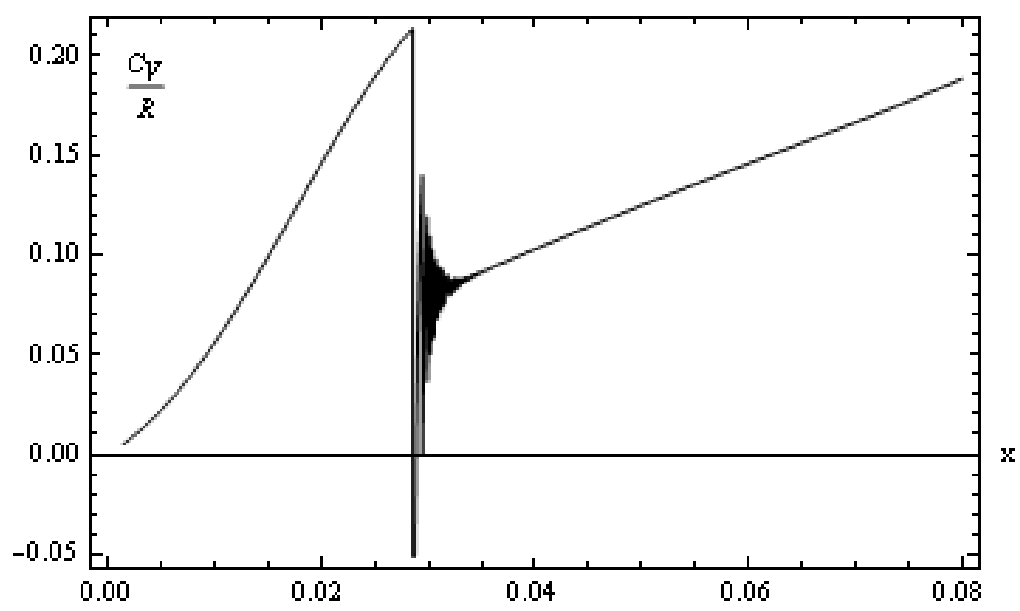

Figure 1. Specific heat calculated with $\mu \approx 34,48276$

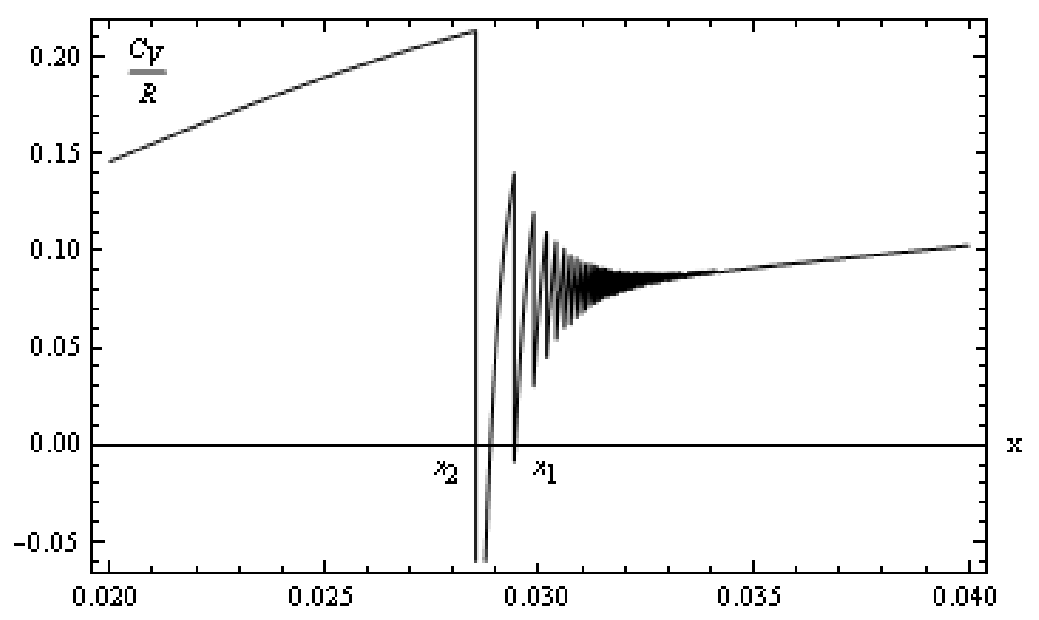

Figure 2. Zoom of specific heat in Fig.1 near the transition phase 